De-Min Wanga ${ }^{\text {, Xin Menga }}$, Xiao-Bin Li, Hao-Jie He, Teng-Fei Zhao, Tian-Wei Jia, Yun He, Yang Yang* and Peng Yu*

\title{
Modification of bovine serum albumin with aminophenylboronic acid as glycan sensor based on surface plasmon resonance and isothermal titration calorimetry
}

DOI 10.1515/hc-2017-0049

Received March 15, 2017; accepted May 30, 2017; previously published online June 24, 2017

\begin{abstract}
Aminophenylboronic acid (ABA) modified bovine serum albumin (BSA) was prepared as neolectin and its interactions with oligosaccharides and glycopolymer were studied by surface plasmon resonance (SPR) and isothermal titration calorimetry (ITC). The conjugation between the primary amine group of the ABA molecule and lysine residues on BSA was performed with an adipate-based strategy to afford the synthetic neoprotein. The number of ABA molecules loaded to BSA surface was determined by matrix-assisted laser desorption/ionization - time of flight (MALDI-TOF) mass spectrometry. In the BSA-ABA and sugar interaction study, no signal was observed for both the SPR and ITC sensor platform using monosaccharides as the analyte, indicating a weak binding affnity, while the galactose modified polymer showed an enhanced response. The binding affinities of the galactosyl-polymer to BSA-ABA from SPR and ITC data were in the micromolar range.
\end{abstract}

a'De-Min Wang and Xin Meng: These authors contributed equally to this work.

*Corresponding authors: Yang Yang and Peng Yu, Key Laboratory of Industrial Fermentation Microbiology of Ministry of Education, Tianjin Key Laboratory of Industry Microbiology, China International Science and Technology Cooperation Base of Food Nutrition/Safety and Medicinal Chemistry, Sino-French Joint Lab of Food Nutrition/ Safety and Medicinal Chemistry, College of Biotechnology, Tianjin University of Science and Technology, Tianjin 300457, China, e-mail: yyang@tust.edu.cn (Y. Yang), yupeng@tust.edu.cn (P. Yu) De-Min Wang, Xin Meng, Xiao-Bin Li, Hao-Jie He, Teng-Fei Zhao and Tian-Wei Jia: Key Laboratory of Industrial Fermentation Microbiology of Ministry of Education, Tianjin Key Laboratory of Industry Microbiology, China International Science and Technology Cooperation Base of Food Nutrition/Safety and Medicinal Chemistry, Sino-French Joint Lab of Food Nutrition/Safety and Medicinal Chemistry, College of Biotechnology, Tianjin University of Science and Technology, Tianjin 300457, China

Yun He: Angstrom Biotechnologies Company, 3350 Scott Blvd., Bldg. 9, Santa Clara, CA 95054, USA
Keywords: aminophenylboronic acid; bovine serum albumin; glycopolymer; isothermal titration calorimetry; surface plasmon resonance.

\section{Introduction}

Glycans, in the form of oligosaccharides, polysaccharides and other glycoconjugates are critical constituents of all living organisms [1-4]. It has been found that the mammalian cell-surface is decorated with a dense and complex array of glycans [5]. Due to their abundance, vast structural heterogeneity and the localization at the outer membrane of cell surface, the carbohydrates as specific recognition sites for various binders, especially proteins, play vital roles in many biological and physiological processes [6] such as intracellular trafficking, cell signaling, proliferation and differentiation, cancer progression, viral and bacterial infection, and immune response [7-11]. Profound understanding of carbohydrate-protein interactions is crucial for the discovery of medicinal, imaging, diagnostic and therapeutic agents [12]. Lectins are a family of carbohydrate-binding proteins that are found in all plants and animals and are one of the major tools widely used in the research for carbohydrate-protein interactions [13, 14]. However, due to the insufficient resources, poor stability, complicated purification and high cost of these commercially available proteins, there is an urgent need to develop alternatives for the carbohydrate "binders" for both pharmaceutical and biomaterial applications [15].

Recently, synthetic small molecules have emerged as carbohydrate-binding lectin mimics [16] for the study of carbohydrate-protein recognition and interaction. Boronic acid (BA) can bind with diols of oligosaccharides under physiological conditions to form boronic acid cyclic diesters in aqueous solution [17]. Thus, polymeric BA tailored macromolecules including silica beads [18], nanoparticles [19-22], magnetic beads [23] and polymers [24, 25], which mimic the multivalent display of lectins on cell 
surface, can be used as artificial sugar receptors to investigate carbohydrate-protein recognition. However, the lack of control over BA presentation on macromolecular backbones makes it difficult to characterize the surface density and spatial distribution of BA after the scaffold modification which limits their further application especially in quantitative analysis of carbohydrate-neolectin interaction.

Herein, we directly attached aminophenylboronic acid (ABA) on the surface of bovine serum albumin (BSA) according to the literature [26] with a different coupling strategy to prepare neolectin (BSA-ABA) for carbohydrate sensor applications, because of the biocompatibility and natural 3D mimetic property of BSA. The multivalent display of ABA can mimic multivalent interactions at carbohydrateprotein interfaces at which avidity is crucial [27-29].
Finally, the number of the small organic molecule on the BSA surface can be easily determined by matrix-assisted laser desorption/ionization - time of flight (MALDI-TOF) mass spectrometry [30]. We also used the BSA-ABA conjugates together with surface plasmon resonance (SPR) and isothermal titration calorimetry (ITC) technology to study the dynamic and thermodynamic details of the carbohydrate-(BSA-ABA) interaction at molecular levels.

\section{Results and discussion}

BSA-ABA conjugates were prepared via covalent coupling between the primary amine of 3-aminophenylboronic acid and lysine residues on BSA by using an adipate NHS ester method [31, 32] (Scheme 1). The adipate linker can make the

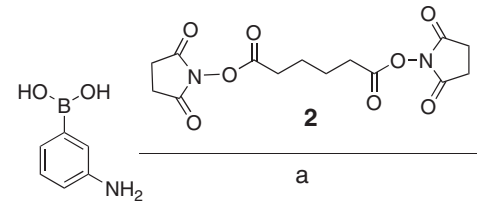<smiles>O=C(CCCCC(=O)ON1C(=O)CCC1=O)Nc1cccc(B(O)O)c1</smiles>

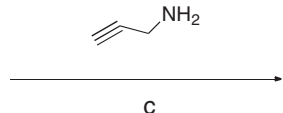

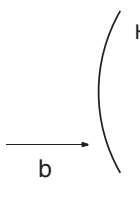

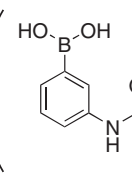<smiles>COC1CC(C)(C)C1CC1C(=O)OC(=O)C1C(C)(C)C</smiles>

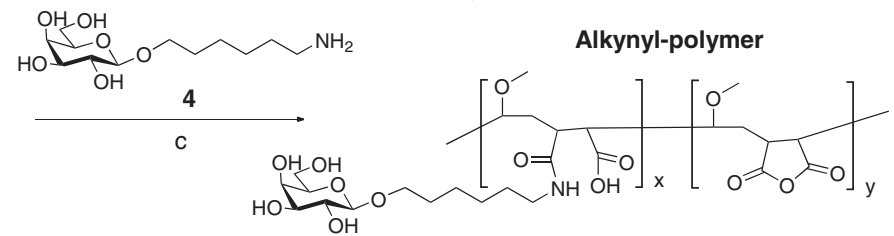

Poly (methyl vinyl ether-alt-maleic anhydride) $\mathrm{MW} \sim 130000$

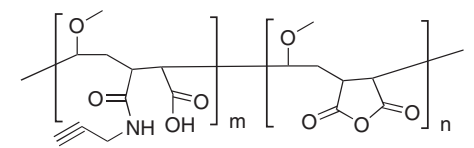

Alkynyl-polymer
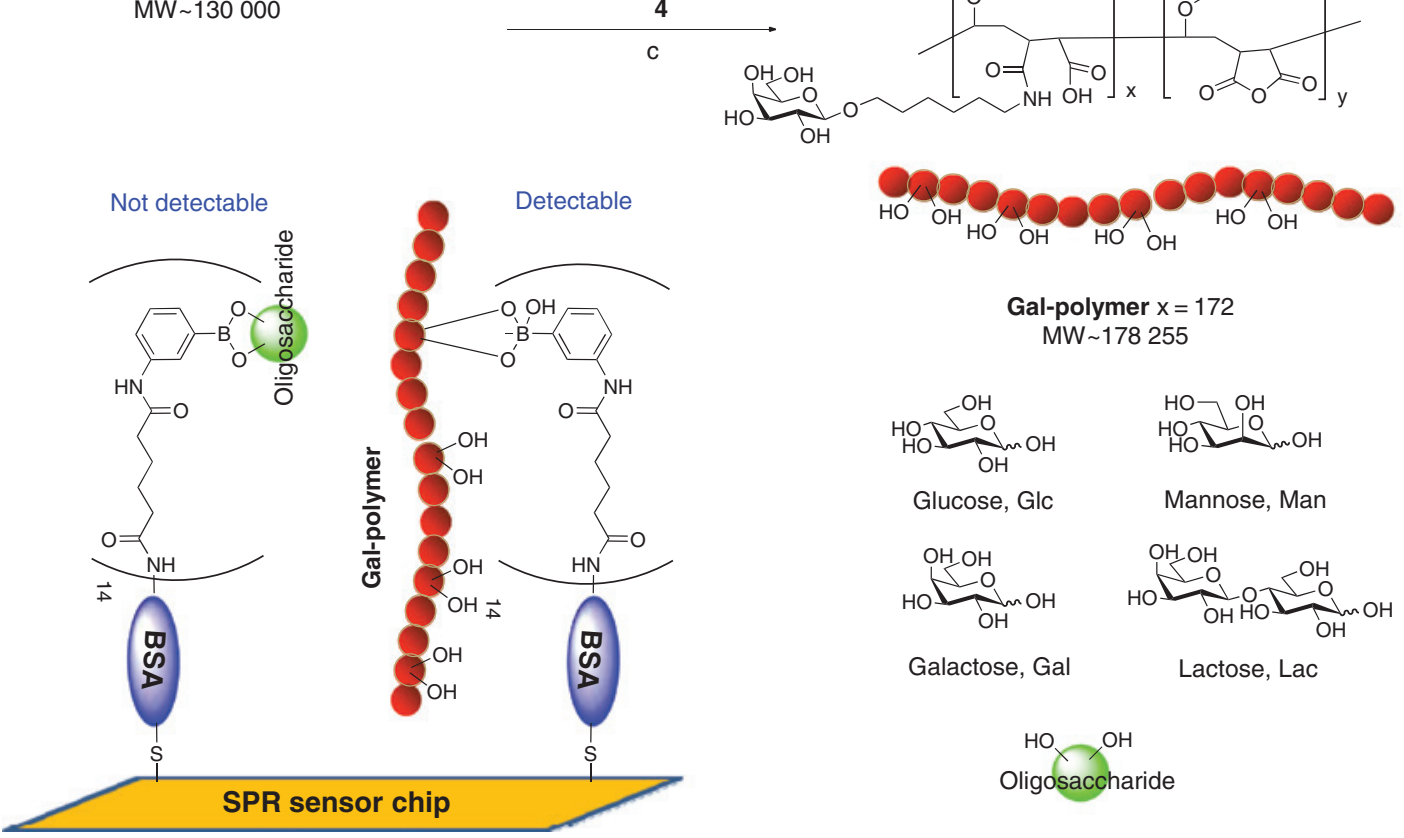

Galactose, Gal

Lactose, Lac

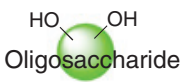

Scheme 1 Preparation of BSA-ABA conjugates and their immobilization onto a SPR chip for biosensor applications. Reagents and conditions: (a) DMSO, Et 3 N, 70\%; (b) PBS, pH 7.4; (c) DMF, room temperature. 
ABA molecule more flexible on the surface of the protein compared with no linker used [26]. It has been reported that cell-surface carbohydrate receptor contains multiple carbohydrate-binding sites and their density regulates the biological events [33]. To mimic the phenomenon of the natural glycan-receptor, various ABA modified BSA conjugates were prepared by changing the ratio of $A B A 3$ to BSA. After dialysis, purification on Sephadex G-75 and lyophilization, the BSA-ABA conjugates were characterized by MALDI-TOF mass spectrometry (see Supplementary Material). Mass analysis of BSA-ABA conjugates showed that an average number of 4,9 or $14 \mathrm{ABA}$ was attached per one molecule of BSA (Table 1).

With the synthetic neolectin in hand, isothermal titration calorimetry (ITC) [34] was first chosen for examining the binding properties of the BSA-ABA conjugates to oligosaccharides, because this technique does not require labeling and immobilization step. Moreover, ITC can determine the enthalpy and the entropy of binding, the association constant $(\mathrm{K})$ and the binding stoichiometry (N) from a single measurement [35]. In the ITC experiment, different sugar (glucose, Glc; mannose, Man; galactose, Gal; lactose, Lac) solutions in a PBS buffer was loaded into the syringe and titrated into the BSA-ABA solution in the calorimeter cell. The heat released at each injection of the PBS buffer to the BSA-ABA was used as a control to correct the titration measurement. To our dismay, trials of thermodynamic evaluation for the interactions between oligosaccharides and BSA-ABA were unsuccessful even when BSA-ABA ${ }_{14}$ the highest ABA density coated on BSA was used, due to the low values of heat in these binding interactions (data not shown). Further increase in the concentration of sugar or BSA-ABA did not significantly enhance the ITC signal and the raw data could not be fitted correctly, indicating that the interaction between BSA-ABA and monosaccharide was very weak. It has been reported that the $\mathrm{K}_{\mathrm{D}}$ value between the boronic acid-functionalized molecularly imprinted polymers (BA-MIPs) and corresponding monosaccharide is typically around a mM-range measured by ITC experiments [36]. We assumed that due to the

Table 1 The conjugations of ABA with BSA.

\begin{tabular}{lrrr}
\hline Entry & Molar ratio & Molecular weight & ABA per BSA \\
\hline BSA & 0 & $66518^{\mathrm{a}}$ & 0 \\
BSA-ABA $_{4}$ & $10 / 1$ & 67600 & 4 \\
BSA-ABA $_{9}$ & $20 / 1$ & 69009 & 9 \\
BSA-ABA $_{14}$ & $30 / 1$ & 70285 & 14 \\
\hline
\end{tabular}

${ }^{\text {a }}$ Molecular weight was determined by MALDI-TOF mass spectrometry. relative low density of the ABA on the BSA surface in our study, the binding affinity was too weak to be measured compared to the high density of BA on BA-MIPs. All the results showed that more sensitive technology should be applied to study such interactions.

Next, another direct-binding measurement on a SPR instrument was established to investigate the dynamic interactions between BSA-ABA and oligosaccharides (Scheme 1). Together with ITC, SPR is also widely accepted as a sensitive and label-free technique for the study of molecular interactions in biological systems [37]. First, the BSA-ABA conjugate was immobilized on the commercial gold chip via gold-thiol covalent coupling in a PBS buffer ( $\mathrm{pH}$ 7.0) [38] at a flow rate of $5 \mu \mathrm{L} / \mathrm{min}$ for channel 2 with the flow of BSA in channel 1 serving as reference. After several minutes for the saturation, different sugars in PBS solution (1 mM) at a flow rate of $30 \mu \mathrm{L} / \mathrm{min}$ for $5 \mathrm{~min}$ (180 $\mathrm{s}$ association phase) were injected over the BSA-ABA coated sensor chip. We observed similar results in the ITC experiments as all sugars exhibited no response signal (data not shown), verifying the low binding affinity of BSA-ABA and its monosaccharide ligands. Similarly, with increasing the concentration of the oligosaccharides to those of the three BSA-ABA conjugates with different ABA densities immobilized on the sensor chip did not result in detectable signals and the dissociation constants could not be determined (data not shown). Similar BSA-ABA conjugates were prepared previously by amidation of carboxylic acid groups in BSA with aminophenyl boronic acid and their sugar binding capacity was also investigated by SPR technique. The affinity constants $\mathrm{K}_{\mathrm{D}}$ were determined to be around a nanomolar range indicating a strong binding between BSA-ABA conjugates and monosaccharides [26]. It can therefore be concluded that higher density of ABA on BSA (maximum 26 ABA per 1 BSA in their system vs 14 ABA per 1 BSA in our study) results in stronger interactions.

Although SPR has received wide acknowledgement for applications for investigation of carbohydrate-protein interaction, the major disadvantage is the difficulty of measuring low molecular mass analytes because of the weak signals, especially when monolayer of proteins is immobilized directly on the flat gold chip sensor surface [39]. Signal enhancement can be achieved successfully using larger molecules, such as nanoparticles [40, 41] and latex particles [42] as backbones for the analytes. These macromolecular scaffolds enhance the signal not only through their high molecular weight but also by the cluster effect [29] for multivalent interactions. It was reported that when polymeric $\mathrm{ABA}$ was immobilized on the gold film of the SPR sensor chip, a selective and 

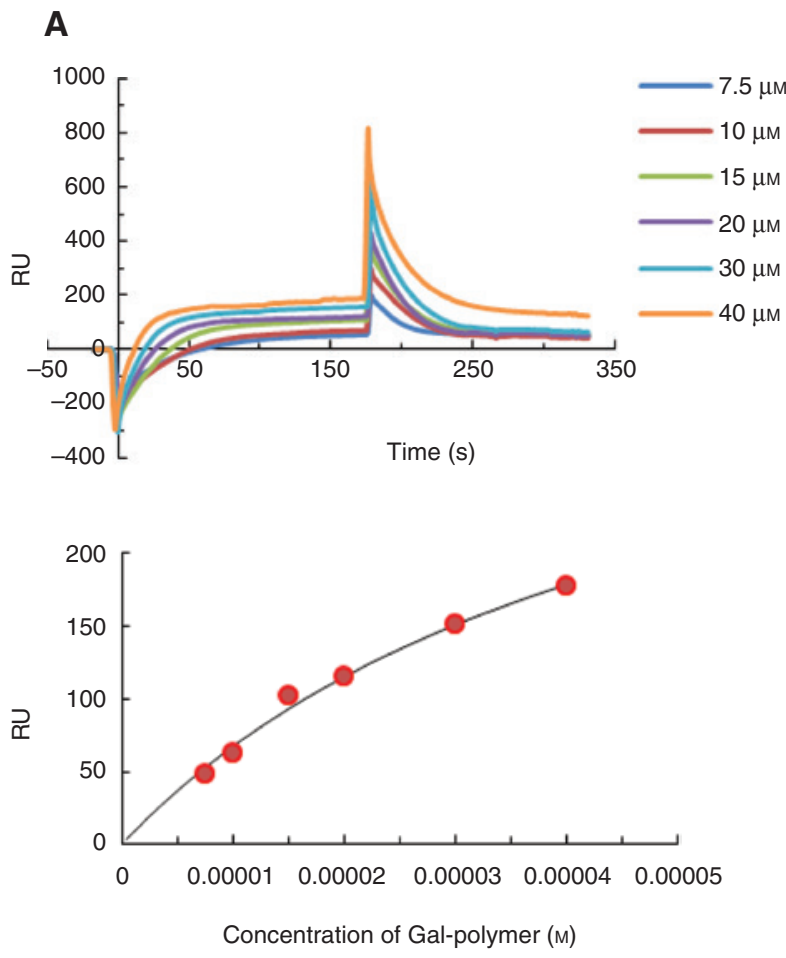

B

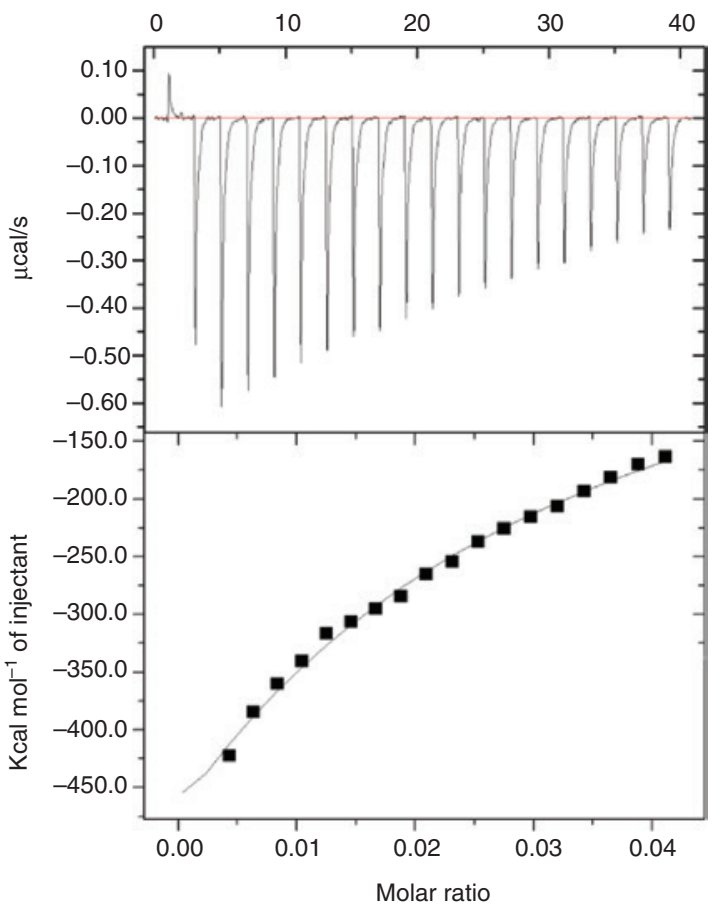

Figure 1 Interaction studies between Gal-polymer and BSA-ABA . $^{\circ}$ (A) SPR sensorgrams and state steady analysis of the binding between Gal-polymer and BSA-ABA ${ }_{14}$ (B) ITC titration curves obtained at $298 \mathrm{~K}$ for the titration of BSA-ABA 14 with Gal-polymer. The top panel shows the raw calorimetric data. Binding parameters were auto-generated after curve fitting using Microcal Origin.

enhanced response to saccharides was observed compared with the self-assembled monolayer presentation of ABA [43]. These results agree with the ITC strategy described above [36]. Inspired by this principle, carbohydrate tailored polymer was chosen in our platform as the analyte to enhance the binding affinity to BSA-ABA coated on the sensor surface.

The synthesis of the galactosyl monomer 4 with an amine group started from peracetylated galactopyranoside, which was obtained using a reported procedure [44] (Scheme 1). Glycopolymer (Gal-polymer) was prepared from [poly(methyl vinyl ether-alt-maleic anhydride), MW 130000 ] by treatment with 4 . The molecular weight of Galpolymer determined by size-exclusion chromatography (SEC) using pullulan as the standard was 178255 which equals to 172 molecules of galactose on the polymer surface. Alkynyl-polymer prepared from polyanhydride by reaction with propargylamine was used as control [45]. The BSA-ABA ${ }_{14}$ was used as the lectin mimic due to its high density of the ABA on the protein surface to investigate the carbohydrate involved interaction (Scheme 1).

All bindings were studied again by SPR and ITC followed by the procedure described above. In the SPR experiment, Gal-polymer binding to immobilized
BSA-ABA $_{14}$ was observed as shown in Figure 1, while a negligible binding was observed when alkynyl-polymer was injected over BSA-ABA ${ }_{14}$. The dissociation constant $\mathrm{k}_{\mathrm{d}}$ and association constant $\mathrm{k}_{\mathrm{a}}$ were determined by the BI-3000 evaluation software (Table 2). Although the thermodynamics constants (entropy and enthalpy) from ITC experiments seemed unreliable, similar binding constants $K_{D}$ in this and the SPR measurement were found. These results can be attributed to the polyvalent interactions between the ABA residues on BSA and the diols of the galactosyl moiety on the Gal-polymer, which monosaccharide does not have.

Table 2 Binding kinetics of alkynyl-polymer and Gal-polymer to $\mathrm{BSA}-\mathrm{ABA}_{14}$ in SPR and ITC assay.

\begin{tabular}{|c|c|c|c|c|}
\hline & \multicolumn{3}{|l|}{ SPR } & \multirow{2}{*}{$\frac{\text { ITC }}{\mathrm{K}_{\mathrm{D}}^{\prime}(\mathrm{M})^{\mathrm{c}}}$} \\
\hline & $K_{A}\left(M^{-1}\right)$ & $K_{D}(M)$ & $\chi^{2}$ & \\
\hline Alkynyl-polymer & $N^{a}$ & NB & NB & NB \\
\hline Gal-polymer & $2.04 \times 10^{4}$ & $4.91 \times 10^{-5}$ & $24.1^{b}$ & $4.61 \times 10^{-6}$ \\
\hline
\end{tabular}

${ }^{\mathrm{a} N B}$ : no binding.

${ }^{\mathrm{b}} \chi^{2}=0.00015 \mathrm{RU}_{\max }^{2} \ll 0.1 \mathrm{RU}_{\max }^{2}$.

' $\mathrm{K}^{\prime}{ }_{\mathrm{D}}$ was calculated as follows: $\Delta \mathrm{G}=-\mathrm{RT} \ln \mathrm{K}=\Delta \mathrm{H}-\mathrm{T} \Delta \mathrm{S}$; $\mathrm{K}_{\mathrm{D}}^{\prime}=1 / \mathrm{K}$. 


\section{Conclusions}

An efficient synthetic strategy was developed for the synthesis of ABA modified BSA as neolectin from lowcost, readily available materials. This convenient method enabled the preparation of a multivalent glycan binder as a promising probe for biological studies. The binding study using SPR and ITC methods indicated that this lectin mimics can be used for the detection of glycopolymer. Further studies on the polysaccharides binding and detection applications of different densities of BSA-ABA are in progress.

\section{Experimental}

The boronic acid-protein conjugates were purified using Sepha$\operatorname{dex}^{\text {TM }}$ G-75 (GE Healthcare). NMR spectra were recorded on a Bruker AVANCE III ( $400 \mathrm{MHz}$ ) instruments. The molecular weights of the aminophenylboronic acid-protein conjugates were confirmed by using a Bruker UltrafleXtreme MALDI-TOF/TOF mass spectrometer. Molecular weights of the polymers were determined by size-exclusion chromatography (SEC) using an Aglient 1200 liquid chromatograph with refractive index detector. All surface plasmon resonance experiments were carried out on a BIAcore 3000 instrument using commercial SPR gold sensor chips (AU 1113 P3, Xantec Bioanalytics $\mathrm{GmbH}$, Germany) at $25^{\circ} \mathrm{C}$. Microcalorimetric experiments were performed with an isothermal titration calorimeter (ITC200). PBS (0.01 M, pH=7.4, without calcium and magnesium) was the running buffer. Solutions of the analytes in buffer were filtered through a $0.22 \mu \mathrm{M}$ micro-membrane and degassed before being injected.

\section{Di-N-succinimidyl adipate (2)}

$\mathrm{N}$-hydroxysuccinimide (5 g, $43 \mathrm{mmol}$ ) and $\mathrm{N}$-ethyl- $\mathrm{N}$-isopropylpropan-2-amine (7.2 mL, $60.5 \mathrm{mmol})$ were dissolved in THF $(100 \mathrm{~mL})$ at $0^{\circ} \mathrm{C}$ and then the solution was slowly treated with adipoyl chloride ( $3 \mathrm{~mL}, 21.5 \mathrm{mmol}$ ). The mixture was stirred for $30 \mathrm{~min}$ and the resultant precipitate was washed with hot isopropanol and dried under reduced pressure to furnish the title product $2(5.9 \mathrm{~g}, 81 \%):{ }^{1} \mathrm{H}$ NMR (400 MHz, DMSO- $\left.d_{6}\right): \delta 2.79(\mathrm{~s}, 8 \mathrm{H}), 2.72(\mathrm{~m}, 4 \mathrm{H}), 1.70(\mathrm{~m}, 4 \mathrm{H})$.

\section{General procedure for BSA-ABA conjugates preparation}

Di- $N$-succinimidyl adipate (2, $248 \mathrm{mg}, 730 \mu \mathrm{mol})$ was dissolved in $1.9 \mathrm{~mL}$ DMSO and trimethylamine $(100 \mu \mathrm{L})$ was added. Aminophenylboronic acid (1, $10 \mathrm{mg}, 73 \mu \mathrm{mol})$ dissolved in $600 \mu \mathrm{L}$ DMSO was pipetted slowly under stirring over $30 \mathrm{~min}$ to the linker solution. After an additional $2 \mathrm{~h}$ of stirring, sodium phosphate buffer ( $0.1 \mathrm{M}$, $\mathrm{pH}$ 7.4) was added. The unreacted linker and DMSO were extracted twice with chloroform. The aqueous phase containing 3 was centrifuged for $1 \mathrm{~min}$ to remove residual $\mathrm{CHCl}_{3}$ and then added to a solution containing BSA in sodium phosphate buffer (10 mL, $0.1 \mathrm{M}, \mathrm{pH} 7.4)$. The mixture was purified on Sephadex G-75 and then freeze-dried. The resulting BSA-ABA conjugate was characterized by MALDI-TOF mass spectrometry and stored at $4^{\circ} \mathrm{C}$ until use.

\section{Polymer preparation}

Amine (100 mg) was dissolved in DMF (10 mL), then poly(methyl vinyl ether-alt-maleic anhydride) $(50 \mathrm{mg}$ ) was added and the solution was slowly stirred at room temperature for $24 \mathrm{~h}$. Then the mixture was dialyzed against deionized water and freeze-dried to yield the Gal-polymer or alkynyl-polymer.

\section{Characterization of glycoconjugates with MALDI-TOF}

The characterization of the BSA-ABA was carried out by matrix assisted laser desorption ionization-time of flight (MALDI-TOF) mass spectrometry in positive linear ion mode using 2,5-dihydroxybenzoic acid (DHB) as matrix. BSA-ABA solutions were diluted with the matrix $(5 \mathrm{mg} / \mathrm{mL}$ matrix in $50 \%$ acetonitrile containing $0.1 \%$ trifluoroacetic acid) at a ratio of 1:5 by volume and deposited onto a MALDI target plate and dried in air. FlexAnalysis was used for analysis of the data.

\section{Surface plasmon resonance analysis}

The interactions between BSA-ABA and Gal-polymer were analyzed at $25^{\circ} \mathrm{C}$ on a BIAcore 3000 instrument. The BSA and BSAABA (1 mM) as immobilized ligands were injected at a flow rate of $5 \mu \mathrm{L} / \mathrm{min}$ on channel 1 and 2, respectively, until saturation was obtained. Gal-polymer analytes were injected over the immobilized BSA-ABA and BSA at flow rate of $30 \mu \mathrm{L} / \mathrm{min}$ for $5 \mathrm{~min}$. The association and dissociation constants of the glycopolymer binding to immobilized BSA-ABA were determined by standard BI 3000 evaluation software.

\section{Isothermal titration calorimetry analysis}

ITC experiments were performed using an ITC200 microcalorimeter in PBS buffer. The concentration of Gal-polymer was $10 \mu \mathrm{M}$, and that of $\mathrm{BSA}^{-\mathrm{ABA}_{14}}$ was $50 \mu \mathrm{m}$. In each individual experiment, $\sim 38 \mu \mathrm{L}$ of Gal-polymer was injected through the computer-controlled $40 \mu \mathrm{L}$ microsyringe at an interval of 2 min into the BSA-ABA solution in the same buffer (cell volume $=200 \mu \mathrm{L}$ ) while stirring at $750 \mathrm{rpm}$. A standard one-site model was fitted to a theoretical titration curve using the software supplied by MicroCal.

Acknowledgements: This work was supported by the Natural Science Foundation of China (21402140, 21502139), the International Science and Technology Cooperation Program of China (2013DFA31160), Key Laboratory of Industrial Fermentation Microbiology of Ministry of Education and Tianjin Key Lab of Industrial Microbiology 
(2015IM107) and Youth Innovation Research Foundation of Tianjin University of Science and Technology (2016LG08).

\section{References}

[1] Bertozzi, C. R.; Kiessling, L. L. Chemical glycobiology. Science. 2001, 291, 2357-2364.

[2] Kiessling, L. L.; Splain, R. A. Chemical approaches to glycobiology. Annu. Rev. Biochem. 2010, 79, 619-653.

[3] Fernandez-Tejada, A.; Javier Canada, F.; Jimenez-Barbero, J. Glycans in medicinal chemistry: an underexploited resource. ChemMedChem. 2015, 10, 1291-1295.

[4] Wang, L.-X.; Davis, B. G. Realizing the promise of chemical glycobiology. Chem. Sci. 2013, 4, 3381-3394.

[5] Varki, A. Evolutionary forces shaping the golgi glycosylation machinery: why cell surface glycans are universal to living cells. Cold Spring Harb. Perspect. Biol. 2011, 3, a005462.

[6] Zeng, X.; Qu, K.; Rehman, A. Glycosylated conductive polymer: a multimodal biointerface for studying carbohydrate-protein interactions. Acc. Chem. Res. 2016, 49, 1624-1633.

[7] Helenius, A.; Aebi, M. Intracellular functions of $\mathrm{N}$-linked glycans. Science. 2001, 291, 2364-2369.

[8] Hart, G. W.; Copeland, R. J. Glycomics hits the big time. Cell. 2010, 143, 672-676.

[9] Marth, J. D.; Grewal, P. K. Mammalian glycosylation in immunity. Nat. Rev. Immunol. 2008, 8, 874-887.

[10] Flitsch, S. L.; Ulijn, R. V. Sugars tied to the spot. Nature. 2003, 421, 219-220.

[11] Karlsson, K. A. Microbial recognition of target-cell glycoconjugates. Curr. Opin. Struct. Biol. 1995, 5, 622-635.

[12] Cheng, Y.; Li, M.; Wang, S.; Peng, H.; Reid, S.; Ni, N.; Fang, H.; Xu, W.; Wang, B. Carbohydrate biomarkers for future disease detection and treatment. Sci. China Chem. 2010, 53, 3-20.

[13] Sakurai, K. Photoaffinity labeling approaches toward identification of carbohydrate-lectin interactions. Trends Glycosci. Glycotechnol. 2015, 27, 1-12.

[14] Wang, B.; Anzai, J.-I. Recent progress in lectin-based biosensors. Materials. 2015, 8, 8590-8607.

[15] Mazik, M. Recent developments in the molecular recognition of carbohydrates by artificial receptors. RSC Adv. 2012, 2, 2630-2642.

[16] Jin, S.; Cheng, Y.; Reid, S.; Li, M.; Wang, B. Carbohydrate recognition by boronolectins, small molecules, and lectins. Med. Res. Rev. 2010, 30, 171-257.

[17] Fang, H.; Kaur, G.; Wang, B. H. Progress in boronic acid-based fluorescent glucose sensors. J. Fluoresc. 2004, 14, 481-489.

[18] Wang, F.; Huang, W.; Guo, C.; Liu, C.-Z. Functionalized magnetic mesoporous silica nanoparticles: Fabrication, laccase adsorption performance and direct laccase capture from Trametes versicolor fermentation broth. Bioresour. Technol. 2012, 126, 117-122.

[19] Bhakta, S.; Seraji, M. S. I.; Suib, S. L.; Rusling, J. F. Antibodylike biorecognition sites for proteins from surface imprinting on nanoparticles. ACS Appl. Mater. Interfaces. 2015, 7, 28197-28206.
[20] Ciftci, H.; Alver, E.; Celik, F.; Metin, A. U.; Tamer, U. Non-enzymatic sensing of glucose using a glassy carbon electrode modified with gold nanoparticles coated with polyethyleneimine and 3-aminophenylboronic acid. Microchim. Acta. 2016, 183, 1479-1486.

[21] Hsieh, H.-H.; Ho, L.-C.; Chang, H.-T. Aminophenylboronic acid polymer nanoparticles for quantitation of glucose and for insulin release. Anal. Bioanal. Chem. 2016, 408, 6557-6565.

[22] Khanal, M.; Barras, A.; Siriwardena, A.; Boukherroub, R.; Szunerits, S. pH-responsive phenylboronic acid-modified diamond particles: Switch in carbohydrate capture ability triggers modulation of physicochemical and lectin-recognition properties. Phys. Status Solidi. A. 2016, 213, 2124-2130.

[23] Pan, M.; Sun, Y.; Zheng, J.; Yang, W. Boronic acid-functionalized core-shell-shell magnetic composite microspheres for the selective enrichment of glycoprotein. ACS Appl. Mater. Interfaces. 2013, 5, 8351-8358.

[24] Chalagalla, S.; Sun, X.-L. Synthesis and characterization of biotin chain-end functionalized boronic acid-containing polymer [boropolymer] as functional glyco-affinity macroligand. React. Funct. Polym. 2010, 70, 471-476.

[25] Zhang, J.; Ni, Y.-L.; Zheng, X.-l. Preparation of poly[vinylphenylboronic acid] chain grafted poly[glycidylmethacrylate-coethylenedimethacrylate] beads for the selective enrichment of glycoprotein. J. Sep. Sci. 2015, 38, 81-86.

[26] Narla, S. N.; Pinnamaneni, P.; Nie, H.; Li, Y.; Sun, X.-L. BSAboronic acid conjugate as lectin mimetics. Biochem. Biophys. Res. Commun. 2014, 443, 562-567.

[27] Cecioni, S.; Imberty, A.; Vidal, S. Glycomimetics versus multivalent glycoconjugates for the design of high affinity lectin ligands. Chem. Rev. 2015, 115, 525-561.

[28] Lee, R. T.; Lee, Y. C. Affinity enhancement by multivalent lectin-carbohydrate interaction. Glycoconj. J. 2000, 17, 543-551.

[29] Lundquist, J. J.; Toone, E. J. The cluster glycoside effect. Chem. Rev. 2002, 102, 555-578.

[30] Yang, Y.; Liu, H.-P.; Yu, Q.; Yang, M.-B.; Wang, D.-M.; Jia, T.-W.; He, H.-J.; He, Y.; Xiao, H.-X.; lyer, S. S.; et al. Multivalent $\mathrm{S}$-sialoside protein conjugates block influenza hemagglutinin and neuraminidase. Carbohydr. Res. 2016, 435, 68-75.

[31] Geissner, A.; Pereira, C. L.; Leddermann, M.; Anish, C.; Seeberger, P. H. Deciphering antigenic determinants of streptococcus pneumoniae serotype 4 capsular polysaccharide using synthetic oligosaccharides. ACS Chem. Biol. 2016, 11, 335-344.

[32] Gruber, W. C.; Scott, D. A.; Emini, E. A. Nyas: development and clinical evaluation of Prevnar 13, a 13-valent pneumocococcal CRM197 conjugate vaccine. In pharmaceutical Science to improve the human condition: Prix Galien 2011. Ann. N.Y. Acad. Sci. 2012, 1263, 15-26.

[33] Horan, N.; Yan, L.; Isobe, H.; Whitesides, G. M.; Kahne, D. Nonstatistical binding of a protein to clustered carbohydrates. Proc. Natl. Acad. Sci. U. S. A. 1999, 96, 11782-11786.

[34] Freyer, M. W.; Lewis, E. A. Isothermal titration calorimetry: experimental design, data analysis, and probing macromolecule/ligand binding and kinetic interactions. Meth. Cell Biol. 2008, 84, 79-113.

[35] Wang, X.; Matei, E.; Gronenborn, A. M.; Ramstrom, O.; Yan, M. Direct measurement of glyconanoparticles and lectin interac- 
tions by isothermal titration calorimetry. Anal. Chem. 2012, 84, 4248-4252.

[36] Awino, J. K.; Gunasekara, R. W.; Zhao, Y. Selective recognition of $d$-aldohexoses in water by boronic acid-functionalized, molecularly imprinted cross-linked micelles. J. Am. Chem. Soc. 2016, 138, 9759-9762.

[37] Singh, P. SPR Biosensors: historical perspectives and current challenges. Sens. Actuators B. 2016, 229, 110-130.

[38] Tao, S.; Jia, T.-W.; Yang, Y.; Chu, L.-Q. BSA-sugar conjugates as ideal building blocks for SPR-based glycan biosensors. ACS Sens. 2017, 2, 57-60.

[39] Pei, R. J.; Yang, X. R.; Wang, E. K. Enhanced surface plasmon resonance immunoassay for human complement factor 4. Anal. Chim. Acta. 2002, 453, 173-179.

[40] Mitchell, J. S.; Wu, Y. Q.; Cook, C. J.; Main, L. Sensitivity enhancement of surface plasmon resonance biosensing of small molecules. Anal. Biochem. 2005, 343, 125-135.

[41] Zeng, S.; Baillargeat, D.; Ho, H.-P.; Yong, K.-T. Nanomaterials enhanced surface plasmon resonance for biological and chemical sensing applications. Chem. Soc. Rev. 2014, 43, 3426-3452.
[42] Severs, A. H.; Schasfoort, R. B. M. Enhanced surface plasmon resonance inhibition test [ESPRIT] using latex particles. Biosens. Bioelectron. 1993, 8, 365-370.

[43] Soh, N.; Sonezaki, M.; Imato, T. Modification of a thin gold film with boronic acid membrane and its application to a saccharide sensor based on surface plasmon resonance. Electroanal. 2003, 15, 1281-1290.

[44] Nagahori, N.; Nishimura, S. I. Tailored glycopolymers: controlling the carbohydrate-protein interaction based on template effect. Biomacromolecules 2001, 2, 22-24.

[45] Zhang, W.-Q.; He, Y.; Yu, Q.; Liu, H.-P.; Wang, D.-M.; Li, X.-B.; Luo, J.; Meng, X.; Qin, H.-J.; Lucchi, N. W.; et al. Polyvalent effect enhances diglycosidic antiplasmodial activity. Eur. J. Med. Chem. 2016, 121, 640-648.

Supplemental Material: The online version of this article (DOI: 10.1515/hc-2017-0049) offers supplementary material, available to authorized users. 